\title{
Postura sentada: a eficácia de um programa de educação para escolares ${ }^{*}$
}

\author{
Seat posture: the efficiency of an educational \\ program for scholars
}

André Rocha Zapater 1

Duani Moraes Silveira 1

Alberto de Vitta 2

Carlos Roberto Padovani 3

José Carlos Plácido da Silva 4

\footnotetext{
1 Curso de Fisioterapia, Departamento de Ciências Biológicas e Profissões da Saúde, Universidade do Sagrado Coração (USC). Rua Irmã Arminda no 10-50, 17011-160, Bauru SP. afdevitta@yahoo.com.br 2 Curso de Fisioterapia e do Programa de PósGraduação em Saúde Coletiva, Universidade do Sagrado Coração (USC), Bauru SP.

3 Instituto de Bioestatística, Universidade Estadual Paulista (UNESP), Botucatu SP.

4 Faculdade de Arquitetura, Artes e Comunicação, Universidade Estadual Paulista (UNESP), Bauru SP. * Projeto financiado pela Fapesp.
}

Abstract This work had as aim the seat posture evaluation program from first-grade students in elementary school, in a public, private and municipal school, in Bauru. For that 71 students were evaluated (pre-test) about knowledges related to seat posture education after all it was given an education program about it, made by exposured class, proof techniques and feedback associated of an effort program supplied by trained professors and finally the revaluation (post-test) of participants knowledge. As a result: 1) on the pretest ocurred valuable difference between schools, the municipal school presented less mistakes number than the private and the public schools; 2) the post-test showed that there was a valuable difference showing that the municipal and private schools have had less mistakes number related to the public; 3 ) in all the schools the mistake situation was bigger before the test than after the test. Comparing schools, according to the mistake situation, when it increased public school has better answers than private schoolt. It's concluded that the program is efficient to increase the school's knowlegde about the seat posture education.

Key words Education program, Seat posture, Scholars and prevention
Resumo Avaliação da eficácia de um programa de educação sobre o conhecimento relacionado à postura sentada de estudantes da 1a série do ensino fundamental, na esfera estadual, particular e municipal, em Bauru. Foram avaliados (pré-teste) 71 alunos e depois aplicado um programa de educação sobre o tema, composto de aulas expositivas, técnicas de demonstração e feedback, associado a um programa de reforço ministrado por professoras treinadas e, finalmente, a reavaliação (pós-teste) dos conhecimentos dos participantes. Foi observado que: 1) no pré-teste ocorreu diferença significativa entre as escolas, sendo que a municipal apresentou menor número de erros do que a particular e a estadual; 2) o pós-teste mostrou que houve diferença significativa sendo que as escolas municipal e particular tiveram um menor número de erros em relação a estadual; 3) em todas as escolas houve predominância da situação de erro pré-teste maior que pós-teste. A escola estadual tem resposta mais freqüente que a particular quando há aumento de erro. Conclui-se que o programa se mostrou eficaz para aumentar os conhecimentos dos escolares a respeito da postura sentada.

Palavras-chave Programa de educação, Postura sentada, Escolares, Prevenção 


\section{Introdução}

No Brasil, de acordo com o Censo Escolar 2000, existem 345.527 escolas no país, sendo 221.852 de ensino fundamental, ensino médio e de educação de jovens e adultos, cujos estudantes se encontram em idades privilegiadas para a formação de valores e hábitos favoráveis à saúde. Das 181.504 escolas do ensino fundamental, 163.368 são da rede pública de ensino. O mesmo ocorre no ensino médio - das 19.456 escolas, 13.227 são públicas - e na educação de jovens e adultos - das 20.892, 18.983 são públicas. Uma ação direcionada para essa rede pública de ensino terá abrangência excepcional do ponto de vista geográfico e populacional. Somente no ano de 2000, 35.717.948 matrículas foram efetivadas no ensino fundamental, 8.192.948, no ensino médio, e 3.140.830, na educação de jovens e adultos. É um público que demanda ações educativas em saúde e merece o desenvolvimento de ações integradas e coesas, para que se alcance sucesso e impacto (Brasil, 2002).

Segundo as Leis de Diretrizes e Bases da Educação (Niskier, 1997), toda criança deverá completar o ensino fundamental. Dessa forma, todo aluno terá de utilizar a postura sentada por, no mínimo, oito anos, cerca de quatro a cinco horas por dia, e de maneira muitas vezes inadequada, $\mathrm{o}$ que já representa um fator de risco para sua saúde, pois, segundo a literatura, é altamente desaconselhável permanecer sentado por mais de 45 a 50 minutos sem interrupções (Paulsen \& Hensen, 1994). Além disso, há a suspeita de que sua reduzida atividade física somada a configurações posturais nem sempre adequadas, tanto em casa como na escola, poderiam provocar um desequilíbrio na sua musculatura, provocando posições anormais de estruturas anatômicas que ainda estão em fase de desenvolvimento.

A postura sentada gera várias alterações nas estruturas músculo-esqueléticas da coluna lombar. O simples fato de o indivíduo passar da postura em pé para a sentada aumenta em aproximadamente $35 \%$ a pressão interna no núcleo do disco intervertebral e todas as estruturas (ligamentos, pequenas articulações e nervos) que ficam na parte posterior são esticadas - isso se o sujeito estiver sentado nas melhores condições possíveis. Além dos problemas lombares, a postura sentada prolongada tende a reduzir a circulação de retorno dos membros inferiores, gerando edema nos pés e tornozelos e, também, promove desconfortos na região do pescoço e membros superiores (Coury, 1994).
Caso o indivíduo sentado realize posturas incorretas por longo período - flexão anterior do tronco, falta de apoio lombar e falta de apoio do antebraço - as alterações são potencializadas, sendo que a pressão intradiscal aumenta para mais de $70 \%$. Este fato pode predispor o indivíduo a maiores índices de desconfortos gerais, tais como dor, sensação de peso e formigamento em diferentes partes do corpo e, principalmente, a processos degenerativos, como a hérnia de disco (Coury, 1994).

Hábitos posturais incorretos, adotados desde o ensino fundamental, são motivos de preocupação. Pelo fato de serem crianças, e não adultos, o esqueleto está em fase de formação, sendo mais susceptível a deformações e as estruturas músculo-esqueléticas, apresentando menor suportabilidade à carga (Knoplich, 1985). É fundamental lembrar que as estruturas que compõem a unidade vertebral (ligamentos e disco intervertebral) sofrem um processo de degeneração ao longo da vida e não possuem mecanismos de regeneração (Rebelatto et al., 1991). Sendo assim, o conjunto de alterações (posturas biomecanicamente incorretas) é fator que potencialmente pode criar condições de prejuízos significativos ao sistema músculoesquelético nos escolares, particularmente às estruturas que compõem a coluna vertebral.

Uma forma de minimizar os efeitos adversos da postura sentada para as estruturas músculo-esqueléticas é o planejamento e/ou replanejamento do ambiente físico, com adoção de mobiliário ajustável a diferentes requisitos da tarefa e às medidas antropométricas individuais. Apenas esta forma de intervenção, contudo, não é suficiente para minimizar o problema. Os programas de treinamentos preventivos são uma outra forma de reduzir sobre o organismo humano os efeitos da postura sentada. De acordo com Stammers \& Patrick (1975), treinamento é o desenvolvimento sistemático de um modelo de habilidade comportamental - atitudes e conhecimento - necessário para promover mudanças no conhecimento e no comportamento dos indivíduos em relação aos seus hábitos e à sua saúde.

Atualmente, as discussões acerca das práticas de treinamentos adotadas são vastas. Uma delas é a utilização de aulas expositivas dialógicas associadas a técnicas de demonstração e feedback. Essa abordagem possibilita a compreensão dos determinantes sociais da educação, porque permite o questionamento, ao mesmo tempo em que proporciona a aquisição de in- 
formações, favorece suas análises críticas, resultando na produção de novos conhecimentos, elimina a relação pedagógica autoritária, valoriza a experiência e conhecimentos prévios dos alunos, estimula o pensamento crítico por meio de questionamentos e problematizações (Lopes, 1995).

A demonstração, técnica também utilizada nos treinamentos, é empregada para ajudar a buscar explicações para os problemas levantados no cotidiano da sala de aula. Permite ao educador aproveitar as experiências trazidas pela criança e proporcionar-lhe uma visão mais elaborada da mesma, o que deverá contribuir para que os alunos busquem soluções para os problemas detectados e estejam habilitados a enfrentar outros problemas ou experiências. Propicia ainda a vivência, a reflexão e a sistematização dos conteúdos tecnológicos e científicos, favorecendo a incorporação das experiências dos alunos às atividades educacionais, tornando-as significativas (Veiga, 1995).

Outro tipo de treinamento preventivo é o modelo "treinando-treinadores". Por meio desse procedimento são treinados indivíduos que posteriormente irão repassar os conhecimentos aos outros. Com relação ao ambiente escolar, especialmente nas séries iniciais, é bastante clara a função do professor como transmissor de idéias e valores. Por essa razão, representa um papel significativo na vida da criança. $\mathrm{O}$ contato diário entre o professor e a criança tende a favorecer e facilitar a assimilação de hábitos e conhecimentos, inclusive aqueles relacionados com o desenvolvimento humano nas diferentes fases da vida, ao funcionamento do corpo humano, alimentação, noções gerais de higiene. E é também capaz de identificar e controlar os aspectos críticos no ambiente escolar - hábitos posturais, mobiliários e movimentos (Oliveira, 1996).

Coury (1998), ao discutir as alternativas de controle para redução de disfunções músculoesqueléticas, relata que um dos grandes desafios dessa temática é o fato de os indivíduos serem altamente refratários a mudanças de hábitos e comportamentos. A mesma autora destaca que um ponto importante relativo ao aspecto educacional é que a idade escolar é uma fase favorável para a instalação de hábitos saudáveis.

Essa constatação reforça a idéia de que é a escola o espaço responsável pela formalização da educação e pelo processo ensino-aprendizagem e que são nos primeiros anos de vida escolar, quando a criança ainda se encontra em fase de crescimento, o melhor momento de iniciar um trabalho de prevenção de problemas músculo-esqueléticos, tornando-os mais eficientes.

Pelos dados e argumentos expostos, o objetivo do presente trabalho foi: avaliar a eficácia de um programa de educação sobre o conhecimento relacionado à postura sentada de estudantes da 1a série do ensino fundamental, de uma escola estadual, particular e municipal, da cidade de Bauru.

\section{Materiais e métodos}

Para a realização do presente trabalho foi utilizado um design quase-experimental com sujeitos únicos, os quais funcionaram como sua própria medida. A variável dependente (critério) foi representada pelos conhecimentos relacionados à postura sentada dos sujeitos, enquanto a variável independente foi o programa de educação.

Foram sujeitos 71 alunos, sendo 26 da escola municipal, 20 da escola particular e 25 da escola estadual, na faixa etária de seis a nove anos, sendo 45 meninos e 26 meninas que cursavam a 1a série do ensino fundamental de uma escola estadual, uma municipal e uma particular, da cidade de Bauru. Localizadas no mesmo bairro, as escolas apresentam o mesmo tipo de mobiliário (mesa e cadeira individual), tendo suas professoras sido treinadas, anteriormente, em uma pesquisa cujo objetivo foi investigar os efeitos de um programa auto-instrucional para identificação e controle de aspectos críticos no ambiente escolar e sobre o conhecimento relacionado à postura sentada em professores da $1 \underline{a}$ série do ensino fundamental, de escolas estaduais, particulares e municipais, da cidade de Bauru.

Os materiais e os equipamentos para a produção, coleta e organização das informações de interesse foram os seguintes: um retroprojetor, folha de questões sobre postura sentada, folha de respostas para as questões, pôsteres sobre o esqueleto humano, sistema muscular e coluna vertebral, transparências ilustrativas com figuras sobre a postura sentada e suas conseqüências, modelo anatômico de um esqueleto humano, filme Músculos e ossos: os alicerces do corpo (Músculos, 1995) e outro sobre os aspectos corretos e incorretos da postura sentada.

O procedimento foi dividido em três etapas.

A primeira foi a realização da avaliação dos conhecimentos sobre postura sentada. Foi apli- 
cado, em todos os estudantes das las séries do ensino fundamental, no início do programa de educação, um questionário com questões fechadas que foram respondidas imediatamente em uma folha à parte. Essas questões abordaram os diversos temas do programa de educação.

Os alunos apenas receberam a folha de respostas, sendo que as questões foram feitas verbalmente pelos pesquisadores. Tal procedimento teve por objetivo garantir que a leitura das questões fosse o mais semelhante possível para todos os participantes e que estes pudessem dar uma resposta rápida, de modo a expressar realmente o que pensavam.

Em seguida foram recolhidas as folhas de respostas para serem utilizadas novamente ao final do programa.

A segunda foi a implementação do programa de educação. Nessa etapa foi proposto um programa de educação com o objetivo de instalar e/ou modificar comportamentos pessoais em relação aos movimentos e posturas corporais inadequadas, capacitando os indivíduos a executar medidas preventivas (autocuidado). O mesmo foi realizado nas escolas durante o horário regular de aulas.

O programa de educação foi composto de duas partes, a primeira delas realizada pelos alunos-pesquisadores, utilizando a modalidade de ensino em grupo, em sessões de duas horas de duração, uma vez por semana, totalizando quatro unidades de ensino. O horário e os dias das aulas foram discutidos com os diretores e professores de cada escola, de modo a alterar minimamente a rotina.

As aulas foram divididas da seguinte forma: 60 minutos de aula expositiva com uso de transparências e pôsteres, com o intuito de informar aos sujeitos sobre o que fazer em relação aos fatores de risco; 35 minutos de técnicas de demonstração e 35 minutos de prática com feedback associada a conseqüências positivas, as quais acrescentaram o como fazer e forneceram subsídios para o desenvolvimento das ações preventivas sobre os fatores de risco relacionados a postura e movimentos.

Os temas foram divididos conforme sugerido por Coury (1994), e podem ser observados no quadro 1.

A segunda parte consistiu de um programa de reforço, em um período de 30 dias durante as aulas regulares ministradas pelas próprias professoras treinadas. Dessa forma, durante o período de realização do programa de educação, o tema discutido pelos alunos-pesquisado- res na semana foi reforçado pelas professoras, que utilizaram as seguintes estratégias facilitadoras propostas pelos pesquisadores: jogo de certo/errado, de sete erros e painéis com figuras ilustrativas sobre as posturas corretas e incorretas.

Finalmente, foi realizada a reavaliação dos conhecimentos sobre postura sentada. Foi aplicado o mesmo instrumento ao final da última aula, seguindo procedimento igual à primeira etapa. O uso de pré e pós-teste, segundo De Vitta (1999), além de permitir a caracterização do nível prévio de informação dessa população sobre o assunto a ser desenvolvido no programa, possibilitou que os próprios participantes identificassem pontos a serem abordados no curso e ficassem alertas para a discussão dos mesmos durante a aula e se auto-avaliassem após o processo de ensino.

O estudo da comparação das freqüências de erros nos diferentes tipos de escolas, considerando as fases (pré e pós-testes) foi realizada utilizando-se a técnica da análise de variância não-paramétrica de Kruskal-Wallis (Siegel \& Castellan, 1988).

Quanto à comparação das porcentagens de erros nas fases (pré e pós-testes) utilizou-se o teste de Goodman para contrastes entre e dentro de proporções multinominais (Goodman, 1964; 1965).

Para a interpretação do resultado do teste estatístico foram utilizadas letras minúsculas e maiúsculas. A leitura da significância do resultado deve ser feita da seguinte maneira: 1) fixando-se a escola, duas proporções seguidas de uma mesma letra maiúscula não diferem ( $\mathrm{p}>$ $0,05)$ quanto às respostas das respectivas situações de erros; 2) fixando-se a situação de erro, duas proporções seguidas de pelo menos uma mesma letra minúscula não diferem quanto às porcentagens de respostas nas correspondentes escolas.

Todas as conclusões estatísticas foram discutidas no nível de 5\% de significância.

\section{Resultados}

Na tabela 1 podem se verificar as medidas descritivas das freqüências de erros no pré-teste segundo escolas e respectivo teste estatístico.

Observa-se que na escola municipal 50\% dos alunos tiveram de $40 \%$ a $73 \%$ de respostas errôneas; na escola particular, $50 \%$ dos alunos tiveram de $53 \%$ a $73 \%$ e na escola estadual, 
Quadro 1

Temas do programa de educação.

1a semana - 1a aula

1. Anatomia básica da coluna vertebral

2. Anatomia básica do membro superior e membro inferior

3. Alguns efeitos possíveis da postura sentada nas costas:

3.1. O que acontece nas costas quando sentamos?

3.2. Possíveis conseqüências da permanência na postura sentada para as costas.

3.3. Como reduzir a sobrecarga que a postura sentada provoca nas costas?

\section{2a semana - 2a aula}

1. Alguns efeitos da postura sentada para as pernas e o pescoço:

1.1. O que acontece em nosso pescoço quando trabalhamos sentados?

1.2. O que acontece em nossas pernas quando trabalhamos sentados?

1.3. Possíveis conseqüências da atividade sedentária para o pescoço.

1.4. Possíveis conseqüências da atividade sedentária para as pernas.

1.5. Como reduzir as posturas extremas e a pouca mobilidade do pescoço durante o trabalho?

3a semana -3 a aula

1. Alguns efeitos possíveis e movimentos dos membros superiores na postura sentada:

1.1. Os membros superiores e a postura sentada.

1.2. Possíveis efeitos de movimentos e posições na postura sentada para os membros superiores.

1.3. Como prevenir os problemas descritos para os membros superiores.

4a semana -4 a aula

1. Uso de microcomputadores: ajustes

Tabela 1

Medidas descritivas das freqüências absoluta e relativa de erros no pré-teste segundo escolas e respectivo teste estatístico.

\begin{tabular}{lcccc}
\hline Medida descritiva & Municipal (26) & $\begin{array}{c}\text { Escola } \\
\text { Particular (20) }\end{array}$ & $\begin{array}{c}\text { Estadual (25) } \\
\text { Resultado do teste estatístico } \\
\text { ("p-value”) }\end{array}$ \\
\hline valor mínimo & $3(20,0 \%)$ & $4(26,6 \%)$ & $5(33,3 \%)$ & \\
1o quartil & $5(33,3 \%)$ & $7(46,6 \%)$ & $7(46,6 \%)$ & \\
mediana & $6(40,0 \%)$ & $8(53,3 \%)$ & $8(53,3 \%)$ & $14,16(\mathrm{p}<0,01)$ \\
3o quartil & $7(46,6 \%)$ & $9(60,0 \%)$ & $10(66,7 \%)$ & \\
valor máximo & $11(73,3 \%)$ & $11(73,3 \%)$ & $14(93,3 \%)$ & \\
\hline
\end{tabular}

$50 \%$ dos alunos erraram de 53\% a $93 \%$ das questões. É possível observar nessa tabela que o teste de Kruskal-Wallis confirma os dados descritivos, ou seja, ocorreu diferença significativa entre as escolas, sendo que a escola municipal apresentou menor número de erros do que as escolas particular e estadual, enquanto estas duas últimas não diferiram entre si.

A tabela 2 mostra as medidas descritivas das freqüências de erros no pós-teste segundo escolas e respectivo teste estatístico.
Nota-se na escola municipal que 50\% dos alunos erraram de $27 \%$ a $53 \%$ das questões; na escola particular, $50 \%$ erraram de $33 \%$ a $53 \%$ das questões e na escola estadual, 50\% dos alunos erraram de $40 \%$ a $73 \%$ das questões. O resultado do teste estatístico de Kruskal-Wallis mostrou que houve diferença estatisticamente significante entre as escolas, sendo que as escolas municipal e particular apresentaram menor número de erros que a escola estadual. 
As porcentagens das situações de erros segundo as escolas estudadas e os resultados do teste estatístico podem ser visualizados na tabela 3 .

Em todas as escolas houve predominância da situação de erro pré-teste maior que pós-teste. Em relação à comparação entre escolas, quanto à situação de erro, verificou-se que no aumento deste, a escola estadual tem resposta mais freqüente que a particular, o contrário ocorrendo quando se considera a diminuição do mesmo.

\section{Discussão}

$\mathrm{Na}$ análise dos resultados relativos às freqüências de erros no pré e pós-testes, verificou-se que o programa educacional promoveu um aumento do conhecimento dos alunos sobre a postura sentada, em todas as escolas estudadas, com resultados estatisticamente significantes.

Alguns estudos demonstram os efeitos de programas educacionais. Santos et al. (1998) aplicaram um programa de educação postural para os escolares, concluindo que o mesmo melhorou o conhecimento sobre desvios pos- turais e suas possíveis causas. De Vitta et al. (2000) realizaram um trabalho para verificar a eficácia de um programa de educação para berçaristas sobre o desenvolvimento motor infantil de crianças de 0 a 2 anos. Verificaram que 93\% dos sujeitos apresentaram mudanças positivas em relação ao conhecimento prévio e $52 \%$ mostraram resultados estatisticamente significantes.

Brassolatti \& Andrade (2002) aplicaram um programa educativo para a prevenção da dengue na cidade de Campinas para os professores de ensino fundamental. Como resultados do pós-teste, verificaram que os participantes obtiveram $100 \%$ de acerto em questões que se referiam ao controle da dengue, sintomas e desenvolvimento da doença e $97 \%$ de acerto na descrição diferencial de adultos do mosquito.

Nogueira et al. (1998) analisaram a eficácia de ações de educação em saúde relativa à hipertensão arterial sistêmica e colesterol em alunos do 1 lo grau, por meio de palestras, e verificaram que houve melhora no nível de conhecimento dos alunos, reforçando a importância da inclusão nas escolas das ações de educação para a saúde.

Tabela 2

Medidas descritivas das freqüências absoluta e relativa de erros no pós-teste segundo escolas e respectivo teste estatístico.

\begin{tabular}{|c|c|c|c|c|}
\hline \multirow[t]{2}{*}{ Medida descritiva } & \multicolumn{3}{|c|}{ Escola } & \multirow{2}{*}{$\begin{array}{l}\text { Resultado do teste estatístico } \\
\text { ("p-value") }\end{array}$} \\
\hline & Municipal (26) & Particular (20) & Estadual (25) & \\
\hline valor mínimo & $1(6,00 \%)$ & $0(0,00 \%)$ & $0(0,00 \%)$ & \\
\hline 1o quartil & $3(20,0 \%)$ & $2(13,3 \%)$ & $4(33,3 \%)$ & \\
\hline mediana & $4(26,6 \%)$ & $4(33,3 \%)$ & $6(40,0 \%)$ & $8,38(\mathrm{p}<0,05)$ \\
\hline 3o quartil & $5(33,3 \%)$ & $7(46,6 \%)$ & $8(53,3 \%)$ & \\
\hline valor máximo & $8(53,3 \%)$ & $8(53,3 \%)$ & $11(73,3 \%)$ & \\
\hline
\end{tabular}

\section{Tabela 3}

Porcentagem da situação de erro segundo escola estudada e resultado do teste estatístico.

\begin{tabular}{lcccc}
\hline Escola & Pré $<$ Pós & $\begin{array}{c}\text { Situação de erro } \\
\text { Pré }=\text { Pós }\end{array}$ & Pré $>$ Pós & Total \\
\hline Municipal & $4(15,38) \mathrm{ab}$ & $1(3,85) \mathrm{a}$ & $21(80,77) \mathrm{ab}$ & 26 \\
Particular & $\mathrm{B}$ & $\mathrm{A}$ & $\mathrm{C}$ & 20 \\
& $0(0,00) \mathrm{a}$ & $1(5,00) \mathrm{a}$ & $19(95,00) \mathrm{b}$ & \\
Estadual & $\mathrm{A}$ & $\mathrm{A}$ & $\mathrm{B}$ & 25 \\
& $6(24,00) \mathrm{b}$ & $3(12,00) \mathrm{a}$ & $16(64,00) \mathrm{a}$ & \\
\hline
\end{tabular}


O aumento do conhecimento gerado pelo programa educacional pode ser explicado por meio de algumas hipóteses, levantadas pelos pesquisadores tais como: a atenção, o interesse, a motivação dos escolares, além dos recursos materiais utilizados. Garcia-Celay \& Tapia (1996) relataram que a atividade educativa torna-se motivante para algumas pessoas e desmotivante para outras. O fato está relacionado aos conteúdos, ao modo como os mesmos são apresentados, ao tipo e à forma de interação do aluno com o professor, aos recursos e às mensagens de que ele se vale. Esses conjuntos de fatores motivam os participantes, facilitando a aprendizagem, pois despertam os interesses.

Segundo Lopes (1995), as aulas expositivas exercem a função de valorizar a vivência do indivíduo, seu conhecimento do concreto, possibilitam o surgimento de dúvidas, problemas e a identificação das respectivas alternativas, auxiliando na reelaboração e na produção do conhecimento. Dessa forma, a intervenção realizada nessa pesquisa, as situações, fatos, idéias, recursos audiovisuais ilustrativos e os modelos anatômicos estimularam a atenção, o interesse e, conseqüentemente, a aprendizagem dos participantes.

Programas educacionais referentes à postura sentada para alunos do ensino fundamental podem produzir mudanças nos conhecimentos e possibilitar que ações preventivas longitudinais possam ser realizadas durante toda a sua vida escolar.

$\mathrm{Na}$ análise dos dados referentes à porcentagem de erros segundo as escolas, observou-se que os alunos da escola particular apresentaram a menor porcentagem de erros no pós-teste em relação ao pré-teste em comparação com as outras duas escolas.

A diferença dos resultados entre as escolas pode ser explicada por questões socioculturais, ambiente familiar, falta de oportunidades, vivências e experiências anteriores, de coordenação de vários sistemas (ou partes) que formam um sistema mais complexo que define nossa individualidade.

Alguns dos estudos sobre a influência sociocultural no desenvolvimento cognitivo das crianças de baixa renda concluem que os baixos índices nos testes de desempenho são provocados pela falta de contato com elementos e padrões culturais próprios das crianças das classes médias e altas. A teoria da privação cultural, que sistematiza esse ponto de vista, explica a deficiência perceptiva atribuída às crianças de baixa renda como resultado de um treinamento visual e auditivo menor do que o oferecido àquelas economicamente mais favorecidas, razão pela qual apresentam níveis de prontidão deficientes na aprendizagem da leitura e da escrita (Montoya, 1996).

Quanto à explicação dos aspectos do ambiente familiar que parecem ter uma influência mais direta e decisiva sobre o desenvolvimento cognitivo, a partir de um conjunto de estudos realizados por outros autores, Montoya (1996) conclui que nos lares das crianças de classe baixa existe uma deficiência de materiais instrutivos (jogos, brinquedos, livros, revistas e objetos que possam ser olhados e manipulados), uma falta de modelos adequados de comportamento fornecidos pelos adultos que cercam as crianças em seus primeiros anos de vida e uma ausência de ajuda efetiva dos pais no sentido de levar a criança a um desenvolvimento perceptivo, verbal e cognitivo adequado.

De uma maneira geral, pode-se supor que as escolas freqüentadas por alunos menos favorecidos apresentam desempenho inferior às de alunos favorecidos, em grande parte, devido às experiências vividas em lares que não transmitem os padrões culturais adequados a um desempenho satisfatório nas tarefas propostas pelos sistemas de avaliação, pela escola e pela sociedade em geral.

Um outro aspecto importante a ser discutido é relativo ao tipo de procedimento educacional aplicado. Nesse estudo foram utilizadas aulas expositivas com feedback e o reforço de professores treinados. Resnicow et al. (1996) relatam que este tipo de procedimento pode ser eficaz em programa de educação postural.

Cardon et al. (2001) aplicaram um programa de cuidados posturais para escolares e investigaram o papel do professor. Compararam três grupos: um grupo com professor treinado, um grupo sem professor treinado e um grupo que não recebeu o programa de intervenção e notaram que o grupo com o professor treinado comparado com os outros dois grupos obteve maior eficácia nos testes práticos e relativos aos conhecimentos. O resultado de uma intervenção educativa na prevenção da dengue indicaram que a participação dos professores possibilitou em parte a diminuição de casos de transmissão nas escolas, pela eliminação de criadouros (Brassolatti \& Andrade, 2002).

O professor treinado é um importante elemento para transferir e reforçar informações sobre as situações de risco do ambiente escolar, 
pois o contato diário dele com o aluno favorece o ensino-aprendizagem, principalmente, se o educador utilizar-se de exercícios que enfatizam a repetição de conceitos e fórmulas, a memorização e que possibilitem, aos escolares, relacionar a teoria com a prática.

De acordo com Balagué et al. (1996), é fundamental incorporar programas de educação postural no currículo de cursos de formação de professores para o ensino fundamental, habilitando-os a implementar no seu dia-a-dia ações preventivas.

As considerações feitas no início deste trabalho, a respeito das vantagens de ações preventivas em saúde na escola e da necessidade de novas alternativas para redução dos efeitos adversos da postura sentada, indicam que a escola de primeiro grau é o espaço adequado para promover programas preventivos relativos a problemas músculo-esqueléticos. No âmbito da educação para a saúde, os dados provenientes desta investigação e de outras que dela decorrerem poderão oferecer contribuições úteis ao planejamento de medidas visando à manutenção, à melhora e à promoção do bem-estar físico dos escolares.
Muitos autores expressam a necessidade de programas educacionais relacionados à saúde, no domínio de saúde escolar, dada a sua capilaridade e abrangência, sendo um aliado importante para a concretização de ações de promoção de saúde para fortalecimento das capacidades dos escolares. Também, nesse campo de estudo, há poucas investigações sistemáticas na literatura nacional e internacional (De Vitta, 1999; Cardon et al., 2001).

O presente trabalho contribuiu para o avanço dessa área de atuação da fisioterapia e proporcionou aumento do conhecimento dos escolares acerca da postura sentada em todas as escolas estudadas. Porém, não é possível confirmar se tais conhecimentos estão sendo aplicados pelos alunos em sua vida diária. Dessa forma, faz-se necessário dar continuidade a essa linha de pesquisa, com estudos que investiguem o comportamento preventivo a longo prazo, com conseqüente mudança de hábito postural, além de programas preventivos que orientem o professor a identificar fatores de risco e atuar no ambiente escolar. 


\section{Referências bibliográficas}

Balagué F, Nordin M, Dutoit G \& Waldburger M 1996. Primary prevention, education, and low back pain among schoolchildren. Bulle Hospital For Joint Diseases 55:130-134.

Brasil. Ministério da Saúde. Secretaria de Políticas de Saúde 2002. A promoção da saúde no contexto escolar. Revista Saúde Pública 36(4):533-535.

Brassolatti RC \& Andrade CFS 2002. Avaliação de uma intervenção educativa na prevenção da dengue. Revista Ciência \& Saúde Coletiva 7(2):243-251.

Cardon G, Bourdeaudhuij ID \& De Clercq D 2001. Back care education in elementary school: a pilot study investigating the complementary role of the class teacher. Patient Education and Counseling 45:219-226.

Coury HJC 1994. Programa auto-instrucional para o controle de desconfortos posturais em indivíduos que trabalham sentados. Dissertação de doutorado. Faculdade de Educação, Universidade Estadual de Campinas, Campinas. 128 pp.

Coury HJC 1998. Self-administered preventive programme for sedentary workers: reducing musculoskeletal symptoms or increasing awareness? Applied Ergonomics 29(6):415-421.

De Vitta FCF, Sanchez FF \& Perez RRM 2000. Desenvolvimento motor infantil: avaliação de um programa de educação para berçaristas. Revista Mimesis 21(1): 1.001-1.118.

De Vitta A 1999. Atuação preventiva em fisioterapia. Edusc, Bauru.

Garcia-Celay IM, Tapia JA 1996. Motivação e aprendizagem escolar, pp. 161-175. In C Coll, J Palacios, A Marchesi. Desenvolvimento psicológico e educação: psicologia da educação. Editora Artes Médicas, Porte Alegre.

Goodman LA 1964. Simultaneous confidence intervals for contrast among multinomial populations. Annals of Mathematical Statistics 35(2):716-725.

Goodman LA 1965. Simultaneous confidence intervals for contrast among multinomial proportions. Technometrics 7(2):247-254.

Knoplich J 1985. A coluna vertebral da criança e do adolescente. Ed. Panamed, São Paulo.
Lopes AO 1995. Aula expositiva: superando o tradicional, pp.35-48. In IPA Veiga (org.). Técnicas de ensino: por que não? Editora Papirus, Campinas.

Montoya AOD 1996. Piaget e a criança favelada: epistemologia genética, diagnóstico e soluções. Ed. Vozes, Petropolis.

Músculos e ossos: os alicerces do corpo 1995. Produção de Bárbara Burst. Direção de Bárbara Burst. [São Paulo]: Vídeos Abril. 1 videocassete (25 min.), VHS, son., color.

Niskier A 1997. LDB: a nova lei da educação. Consultor, Rio de Janeiro.

Nogueira et al. 1998. Hipertensão arterial e colesterol: proposta educativa. Revista Ciência Farmacêutica 1(19):77-92.

Oliveira RJP 1996. Postura de crianças em sala de aula: um estudo diagnóstico. Dissertação de mestrado. Programa de Pós-Graduação em Educação, Universidade Federal de São Carlos, São Carlos.

Paulsen AS \& Hensen JA 1994. The working positions of schoolchildren. Applied Ergonomics 25(1):63-64.

Rebelatto JR, Caldas MAJ \& De Vitta A 1991. Influência do transporte do material escolar sobre a ocorrência de desvios posturais em estudantes. Revista Brasileira de Ortopedia 26(11-12):403-410.

Resnicow K, Robinson TN \& Frank E 1996. Advances and future directions for school-based health promotion research: commentary on the $\mathrm{CATCH}$ intervention trial. Preventive Medicine 25:378-83.

Santos SG et al. 1998. Educação postural mediante um trabalho teórico. Revista Brasileira de Atividade Física \& Saúde 3(2):32-42.

Siegel S \& Castellan JRNJ 1988. Nonparametric statistics for the behavioral sciences. Ed. Mc Graw-Hill, Nova York.

Stammers R \& Patrick J 1975. The psychology of training. Ed. Methuen, Londres.

Veiga IPA 1995. Nos laboratórios e oficinas escolares: a demonstração didática, pp. 131-147. In IPA Veiga (org.). Técnicas de ensino: por que não? Editora Papirus, Campinas.

Artigo apresentado em 30/5/2003

Aprovado em 7/7/2003

Versão final apresentada em 14/10/2003 